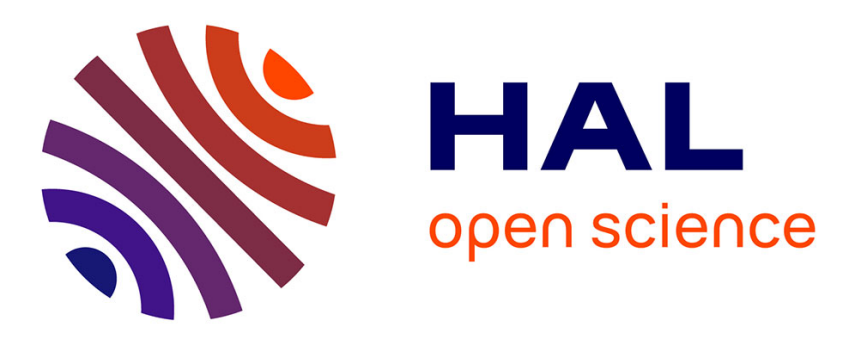

\title{
Mapping fluxes of radicals from the combination of electrochemical activation and optical microscopy
}

Sorin Munteanu, Jean Roger, Yasmine Fedala, Fabien Amiot, C Combellas,

Gilles Tessier, Frédéric Kanoufi

\section{- To cite this version:}

Sorin Munteanu, Jean Roger, Yasmine Fedala, Fabien Amiot, C Combellas, et al.. Mapping fluxes of radicals from the combination of electrochemical activation and optical microscopy. Faraday Discussions, 2013, 164 (1), pp.241 - 258. 10.1039/C3FD00024A . hal-02300154

\section{HAL Id: hal-02300154 \\ https://hal.science/hal-02300154}

Submitted on 29 Sep 2019

HAL is a multi-disciplinary open access archive for the deposit and dissemination of scientific research documents, whether they are published or not. The documents may come from teaching and research institutions in France or abroad, or from public or private research centers.
L'archive ouverte pluridisciplinaire $\mathbf{H A L}$, est destinée au dépôt et à la diffusion de documents scientifiques de niveau recherche, publiés ou non, émanant des établissements d'enseignement et de recherche français ou étrangers, des laboratoires publics ou privés. 


\title{
Mapping fluxes of radicals from the combination of electrochemical activation and optical microscopy
}

\author{
Sorin Munteanu, ${ }^{\mathrm{a}}$ Jean Paul Roger, ${ }^{\mathrm{b}}$ Yasmina Fedala, ${ }^{\mathrm{a}, \mathrm{b}}$ Fabien Amiot, ${ }^{\mathrm{c}}$ Catherine Combellas, ${ }^{\mathrm{a}}$ \\ Gilles Tessier ${ }^{b}$ and Frédéric Kanoufi, ${ }^{a, *}$
}

\author{
${ }^{a}$ CNRS UMR7195, ESPCI ParisTech, 10 rue Vauquelin, 75231 Paris Cedex 05, France. Tel: +33 14079 4526; E-mail: \\ frederic.kanoufi@espci.fr \\ ${ }^{b}$ Institut Langevin CNRS UMR 7587, ESPCI ParisTech, 1 rue Jussieu, 75238 Paris Cedex 05, France, \\ ${ }^{c}$ FEMTO-ST Institute, CNRS-UMR 6174/UFC/ENSMM/UTBM, 24 chemin de l'Épitaphe, 25030 Besançon, France
}

\begin{abstract}
The coating of gold $(\mathrm{Au})$ electrode surfaces with nitrophenyl (NP) layers is studied by combination of electrochemical actuation and optical detection. The electrochemical actuation of the reduction of the nitrobenzenediazonium (NBD) precursor is used to generate NP radicals and therefore initiate the electrografting. The electrografting process is followed in situ and in real time by light reflectivity microscopy imaging, allowing for spatio-temporal imaging with sub-micrometric lateral resolution and sub-nanometer thickness sensitivity of the local growth of a transparent organic coating onto a reflecting Au electrode. The interest of the electrochemical actuation resides in its ability to finely control the grafting rate of the NP layer through the electrode potential. Coupling the electrochemical actuation with microscopic imaging of the electrode surface allows quantitative estimate of the local grafting rates and subsequently a real time and in situ mapping of the reacting fluxes of NP radicals on the surface. Over the 2 orders of magnitude range of grafting rates (from 0.04 to $4 \mathrm{~nm} / \mathrm{s}$ ), it is demonstrated that the edge of $\mathrm{Au}$ electrodes are grafted $\sim 1.3$ times more quickly than their centre, illustrating the manifestation of edge-effects on flux distribution at an electrode. A model is proposed to explain the observed edge-effect, it relies on the short lifetime of the intermediate NP radical species.
\end{abstract}

\section{Introduction}

Monitoring the chemical transformation of surfaces is of considerable importance in many fields and related applications going from biology, catalysis, electronics or energy. The most promising approaches to monitor the chemical reactivity of surfaces rely on high-resolution spatio-temporal inspection. Mapping the local chemical reactivity of surfaces is possible using real "chemical" microscopes, allowing for the micrometric observation of local chemical reactions. As such, the scanning electrochemical microscope (SECM) has been proposed since the early 1990s as a true scanning "chemical" probe microscope allowing for in situ imaging of surface chemical processes. More recently, imaging optical microscopes have emerged in chemical sciences, allowing one to optically image surface transformation processes. ${ }^{1}$ If they provide a full-field view of surface transformations, they usually require dedicated labelling species as in the numerous fluorescence microscopies, ${ }^{2-4}$ or surface enhanced Raman spectroscopy. ${ }^{5-8}$ Alternatively, label-free approaches, such as those provided by surface plasmon resonance ${ }^{9,10}$ or ellipsometry ${ }^{11}$ microscopies, provide the imaging of the local change in the refractive index associated to the chemical transformation of an interface.

To map the chemical transformation of a surface, the next requisite is to develop strategies to direct such transformation during the local inspection of the surface. In this respect, electrochemistry or an electrochemical activation offer promising potentialities. Indeed, the electrochemical activation of an electrode surface allows for the minute generation of gradients of chemical reagents concentrations.

Combining electrochemical activation of chemical reagent gradients with chemical microscopy surface imaging then provides a promising strategy to modulate and visualise the spatio-temporal repartition of the chemical reactivity of surfaces. It has been illustrated in many aspects by SECM for the controlled local etching of surfaces ${ }^{12-14}$ or in more recent examples for local surface reactivity mappings. ${ }^{15,16}$ The observation of electrochemically generated chemical gradients by optical microscopies has also been described. ${ }^{17-19}$ In seminal works, electrochemical activation was used to activate either gradients of concentrated highly absorbing species $^{17}$ or a fluorescent probe by electrochemiluminescence, ${ }^{4,20-23}$ allowing one to map the electrochemically-driven fluxes of the electrogenerated species at the electrode. More recently, label-free approaches have been proposed and SPR microscopy was used to analyse local traces of explosives on fingerprints decorated surfaces. ${ }^{9}$ Our group has developed a versatile alternative approach using ellipsometric microscopy to monitor in situ and in real-time the chemical transformation of surfaces by organic layers. ${ }^{11}$

Here, we exploit the combination of such opto-electrochemical microscopies to visualise and map the reaction of electrogenerated gradients of radicals with an electrode surface. Indeed, the electrochemical reductive activation of an 
electrode in an aryldiazonium solution allows for the generation of phenyl radical species in the vicinity of the electrode. These reactive species are then prone to react with their generating surface and the deposition of a thin organic aryl multilayer (several nanometres thick) on the electrode ensues. Even though phenyl radicals are highly reactive, their reaction with a surface is rather slow, as attested by the slow growth rate of the organic layer. The rate of deposition of the organic layer on the electrode is then an indirect measure of the flux of radicals reacting with the surface. Visualising and mapping the spatio-temporal growth of an aryl multilayer from an electrode surface then provides an access to the mapping of the reactivity of phenyl radicals with the surface.

The present report aims at inspecting and mapping the reactivity of nitrophenyl (NP) radicals at surfaces. As illustrated in Figure 1, NP radicals are generated at different gold $(\mathrm{Au})$ microelectrodes from reduction of nitrobenzenediazonium (NBD), while the extent of the local reaction of NP radicals with the Au surface is monitored by light reflectivity microscopy. ${ }^{24}$ After a brief description of the optical method, its performances are tested through its coupling with the electrochemical activation of the surface for the inspection of local fluxes of electrogenerated reactive radicals.

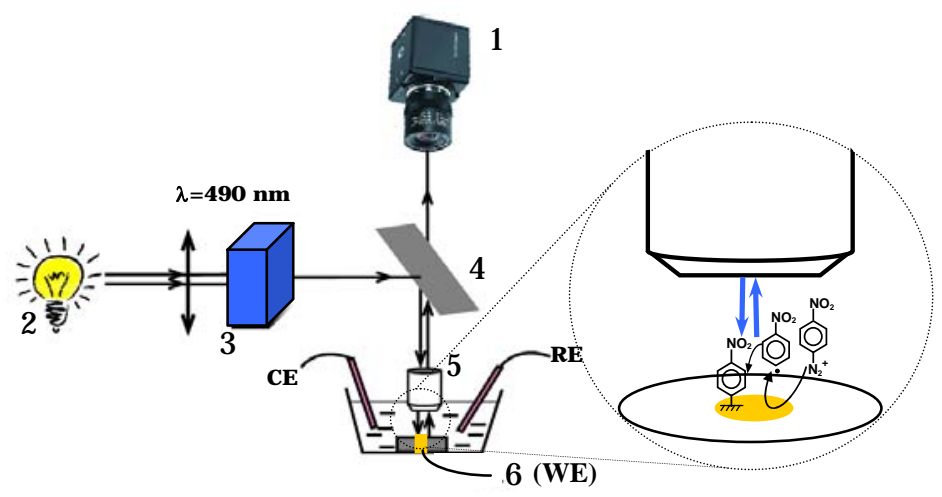

Fig. 1 Schematic representation of light reflectivity mapping of the grafting of nitrophenyl (NP) radical onto Au microelectrodes under electroreduction of nitrobenzenediazonium (NBD) solution.

\section{Experimental Part}

Materials. Nitrobenzendiazonium (NBD) acetonitrile $(\mathrm{ACN})$, tetrabutylammonium tetrafluoroborate $\left(\mathrm{NBu}_{4} \mathrm{BF}_{4}\right)$ from Sigma-Aldrich were used as received. Macroscopic gold $(\mathrm{Au})$ electrodes were plates cut in Au coated silicon wafer obtained from Sigma-Aldrich (100 nm coating). Gold microelectrodes were home-made from Au wires $(25$ or $250 \mu \mathrm{m}$ diameter, Goodfellow) sealed in borosilicate glass capillaries (Sutter).

The electrografting of the different $\mathrm{Au}$ surfaces was performed at room temperature, $20^{\circ} \mathrm{C}$, in $5 \mathrm{mM} \mathrm{NBD}+0.1 \mathrm{M}$ $\mathrm{NBu}_{4} \mathrm{BF}_{4} \mathrm{ACN}$ solution by chronoamperometric experiments in a three-electrode configuration (counter electrode, $\mathrm{CE}$ : $1 \mathrm{~mm}$ diameter $\mathrm{Pt}$ wire, and quasi-reference, RE: $1 \mathrm{~mm}$ diameter $\mathrm{Ag}$ wire anodized) using a $\mathrm{CH}$ 660C potentiostat $(\mathrm{CH}$ Instruments).

The light reflectivity microscopic observation schematized in Figure 1 was obtained using a standard microscope (Olympus), equipped with a camera (1, Dalsa 1M30). The light of a halogen white lamp (2), spectrally filtered (3) to obtain a blue illumination $(\lambda=490 \mathrm{~nm}, \Delta \lambda=20 \mathrm{~nm})$, is directed towards the sample by an optical separator (4). The substrate (6) is therefore illuminated from the top by a light beam with a known numerical aperture at zero mean incidence via a microscope objective (5). The reflected light is collected by the same objective and sent on the CCD camera (1), which allows real time imaging of the light flux reflected by the analyzed surface. The incident light intensity is chosen so that the reflected light reaching the camera detector is close but smaller than saturation level. It corresponds to an incident light beam of measured radiant power of $170 \mu \mathrm{W}$ which illuminates a $3 \mathrm{~mm}$ diameter spot of the studied substrate (light flux density of $2.4 \mathrm{~mW} \mathrm{~cm}^{-2}$ ). The light intensity is so low that the illumination cannot heat the substrate/solution by more than $10^{-6}{ }^{\circ} \mathrm{C}$.

The first step of the observation procedure consists in the precise adjustment of the illumination incidence on the surface. This is achieved with an accuracy of $10^{-2} \circ$, in air byusing a Mirau interferential objective (Nikon). The electrolytic solution is then poured into the electrochemical cell in which in situ observation is obtained with a waterimmersion objective $(10 \mathrm{x}, \mathrm{NA}=0.3, \mathrm{WD}=3.3 \mathrm{~mm}$, or $40 \mathrm{x}, \mathrm{NA}=0.80, \mathrm{WD}=3.3 \mathrm{~mm})$. Images of the reflecting surface are captured with a CCD camera interfaced with a home-programmed Labview program. Each acquired image is a stack of 6 snapshots, each integrated over $50 \mathrm{~ms}$, for a total duration of $300 \mathrm{~ms}$. Image analysis is achieved using Matlab routines.

\section{A. Principle of Light Reflectivity Microscopy}

The principle of the method is based on monitoring the surface reflectivity variations by simply measuring the 
intensity of light that has been reflected by the illuminated reflective surface. The light reflectivity, $R$, is defined as the ratio of the intensity of the reflected beam, $I_{\text {refl }}$, to the intensity of the incident beam, $I_{\text {inc }}: R=I_{\text {refl }} / I_{\text {inc }}$. Under normal incidence, as obtained in our set-up, the expression of the light reflectivity of a beam propagating in a transparent ambient medium (medium A, real refractive index $n_{A}$ ) reflecting on an opaque substrate (medium $S$ ), is given by (1).

$$
\mathrm{R}_{\mathrm{AS}}=\left|\tilde{\mathrm{r}}_{\mathrm{AS}}\right|^{2}=\left|\frac{\mathrm{n}_{\mathrm{A}}-\tilde{\mathrm{n}}_{\mathrm{S}}}{\mathrm{n}_{\mathrm{A}}+\tilde{\mathrm{n}}_{\mathrm{S}}}\right|^{2}
$$

The substrate is optically characterized by a complex refractive index $\tilde{n}_{S}=n_{S}+i k_{S}$, where $n_{S}$ represents the real part of the refractive index, and $\mathrm{k}_{\mathrm{S}}$ is the imaginary part, related to the extinction coefficient. ${ }^{25}$

The light reflectivity is equal to the square of the modulus of the reflection coefficient of an electromagnetic wave, $\tilde{\mathrm{r}}_{\mathrm{AS}}$, which is a complex number obtained through Fresnel equations.

When the light passes through an intermediate organic layer (medium $\mathrm{L}$ with refractive index $\mathrm{n}_{\mathrm{L}} \approx 1.5$, and thickness th) between the ambient medium and the reflecting substrate, the reflection coefficients at each interface (ambient/layer interface, $\mathrm{A} / \mathrm{L}$ and layer/substrate interface, $\mathrm{L} / \mathrm{S}$ ) can be expressed by relations similar to (1). The overall light reflectivity takes into consideration the multiple reflections at each interface $\left(\mathrm{A} / \mathrm{L}\right.$ described by $\tilde{\mathrm{r}}_{\mathrm{AL}}$ and $\mathrm{L} / \mathrm{S}$ described by $\tilde{\mathrm{r}}_{\mathrm{LS}}$ ), along with the phase shift induced in the deposited layer. The expression of light reflectivity extends to: ${ }^{26}$

$$
\mathrm{R}=|\tilde{\mathbf{r}}|^{2}=\left|\frac{\tilde{\mathrm{r}}_{\mathrm{AL}}+\tilde{\mathrm{r}}_{\mathrm{LS}} \mathrm{e}^{2 \mathrm{i} \frac{2 \pi}{\lambda} \mathrm{n}_{\mathrm{L}} \text { th }}}{1+\tilde{\mathrm{r}}_{\mathrm{AL}} \tilde{\mathrm{r}}_{\mathrm{LS}} \mathrm{e}^{2 \mathrm{i} \frac{2 \pi}{\lambda} \mathrm{n}_{\mathrm{L}} \mathrm{th}}}\right|^{2}
$$

If the incident light beam has a constant intensity, one can measure, from recorded snapshots, the evolution of the reflected beam light intensity collected at time $t$, compared to the one recorded at time $t=0$, which is related to the relative variation of reflectivity:

$$
\frac{\mathrm{I}_{\mathrm{refl}}(\mathrm{t})}{\mathrm{I}_{\mathrm{refl}}(0)}=1+\frac{\Delta \mathrm{R}}{\mathrm{R}}
$$

Thus, a continuous acquisition of images allows one to determine the local evolution of a thin layer deposition on a reflecting surface.

The sensitivity of the technique is directly dependent on the optical properties of the studied surface (optical indices of the substrate, and of the deposited thin layer to be analyzed). If the refractive indices are known, the thickness th can be deduced from optical measurements for low thicknesses. For thick films, full knowledge of the evolution of the reflectivity is necessary in order to resolve ambiguities introduced by the periodicity of (2).

Gold is a particularly appealing substrate for light reflectivity measurements and high sensitivity is obtained for blue light illumination $(\lambda<500 \mathrm{~nm})$. The theoretical relative variations of light reflectivity on an Au surface $\left(\tilde{\mathrm{n}}_{\mathrm{S}}=1.02+\right.$ $1.83 \mathrm{i}$ for $\lambda=490 \mathrm{~nm}),{ }^{27}$ covered by an homogeneous organic layer (thickness th and refractive index $\mathrm{n}_{\mathrm{L}}=1.5$ ), for a light beam $(\lambda=490 \mathrm{~nm})$ propagating in $\mathrm{ACN}\left(\mathrm{n}_{\mathrm{A}}=1.34\right)$ can be obtained from (2). The relative variations of light reflectivity that are presented in Figure 2 show periodic oscillations with the layer thickness with a period in thickness of $\Delta \mathrm{th}=\lambda / 2 \mathrm{n}_{\mathrm{L}}\left(\Delta \mathrm{th}=163 \mathrm{~nm}\right.$ for $\lambda=490 \mathrm{~nm}$ and $\left.\mathrm{n}_{\mathrm{L}}=1.5\right)$. For a thin layer deposition, $\mathrm{th}<30 \mathrm{~nm}$, the relative light reflectivity change decreases linearly with the deposited layer thickness, th, according to:

$$
\frac{\Delta \mathrm{R}}{\mathrm{R}}=-\sigma \text { th } \quad(\text { for } \mathrm{th}<30 \mathrm{~nm})
$$

with $\sigma$ the sensitivity of the optical method, $\sigma=0.0043 \mathrm{~nm}^{-1}$ for $\lambda=490 \mathrm{~nm}$ and $\mathrm{n}_{\mathrm{L}}=1.5$ and for measurements performed in a liquid ambient medium $\left(\mathrm{n}_{\mathrm{A}}=1.34\right)$. This sensitivity is much higher than that expected for electroreflectance, the change of light reflectivity associated to the change of refractive index due to potential activation of the double layer at the electrode-electrolyte interface ${ }^{2428}$ Owing to the good stability of the light source, the noise level or light drift induces a limit of detection for the variations of $1+\Delta R / R<0.002$. 


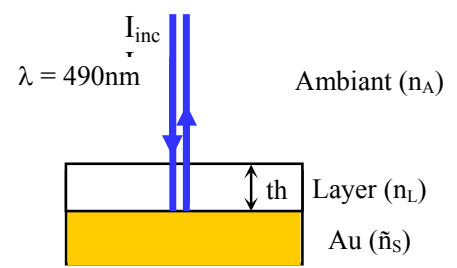

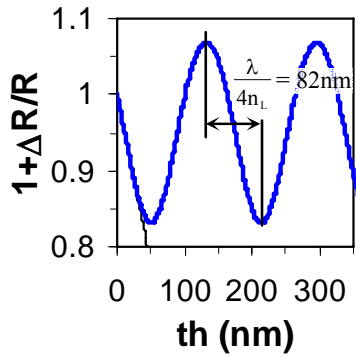

Fig. 2 Variation of reflectivity of light propagated in an ambient medium at an Au surface coated with a transparent layer of thickness th and refractive index $n_{L}$. a) optical model; b) theoretical relative variation of the light reflectivity with the layer thickness for a liquid ambient ( $\mathrm{n}_{\mathrm{A}}$ $=1.34), \tilde{\mathrm{n}}_{\mathrm{S}}=1.02+1.83 \mathrm{i}$ for $\lambda=490 \mathrm{~nm}$, and $\mathrm{n}_{\mathrm{L}}=1.5$.

This work is devoted to the mapping of fluxes of NP radical species from the mapping of the local deposition of thin NP multilayers. Indeed, the growth rate of the thin layer is related to the flux of incoming and reacting NP radicals. It could then be easier to express the relative light reflectivity variation in term of change in surfacic concentration of the deposited material, $\Gamma$ (in $\mathrm{mol} / \mathrm{cm}^{2}$ ), which can be obtained from the molecular mass of the deposited moiety, M, and the density of the formed thin layer, $\rho$ :

$$
\text { th }=\frac{M}{\rho} \Gamma
$$

In the approximation of a thin layer deposition, the relative light reflectivity change also decreases linearly with the deposited material surface concentration and (4) becomes:

$$
\frac{\Delta \mathrm{R}}{\mathrm{R}}=-\sigma \frac{\mathrm{M}}{\rho} \text { th } \quad(\text { for } \mathrm{th}<30 \mathrm{~nm})
$$

The latter expression allows one to express more easily layer growth rates in units of molecular fluxes in mol cm $\mathrm{cm}^{-1}$.

\section{B. Results and Discussion}

\section{Inspecting nitrophenyl radical reaction on macroscopic electrodes}

First, we illustrate briefly the potentiality of light reflectivity microscopy for the real time and in situ monitoring of the electrografting of a macroscopic Au wafer surface by the NBD salt. Figure 3a presents an example of a snapshot of a $850 \times 850 \mu \mathrm{m}^{2}$ area of a $\mathrm{Au}$ surface (total area $0.7 \times 0.7 \mathrm{~cm}^{2}$ ) in a $5 \mathrm{mM}$ NBD solution before the electrochemical activation. After $10 \mathrm{~s}$, the electrode grafting is initiated by potential activation at a constant $\mathrm{E}_{\mathrm{Au}}=-0.4 \mathrm{~V} \mathrm{vs} \mathrm{Ag} / \mathrm{AgCl}$, while snapshots are continuously acquired every $2 \mathrm{~s}$. The last snapshot taken at the end of the electrografting is then transcribed from eq (3) into a local variation of light reflectivity at each CCD camera pixel of coordinate (x,y); for that, the reflected light intensity at each pixel $I_{r e f l}(x, y, t)$ is compared to that obtained on the same pixel in the initial image, $\mathrm{I}_{\mathrm{refl}}(\mathrm{x}, \mathrm{y}, \mathrm{t}=0)$. The local variation of light reflectivity on the inspected region of the Au macroelectrode is given in Figure $3 \mathrm{~b}$. As predicted from the optical model presented in section A, the electrografting procedure results in the decrease of the intensity of the light reflected by the surface.

The time-evolution of the overall reflectivity change (Figure $3 \mathrm{c}$ ), $1+\Delta \mathrm{R} / \mathrm{R}$, averaged over a $10 \times 10 \mu \mathrm{m}^{2}$ area of the imaged macroelectrode is transcribed, using eq. (4), into the time-evolution of the thickness, th(t), of the NP layer. It is presented in Figure 3d and paralleled with the evolution of the charge, Q(t) passed in the whole macroscopic electrode during the electrografting process. The growth of the coating layer can be described by two characteristic timescales. During a first short induction period $<4-6 \mathrm{~s}$, the layer grows quickly while a large amount of charge is quickly exchanged. After this induction period, both the layer thickness and the injected charge steadily increase. The steady layer thickness growth is characterized by a growth rate,

$$
v=\frac{\mathrm{d} \text { th }}{\mathrm{dt}}
$$

estimated from the reflectivity measurement and eq (4) as $v=0.016 \mathrm{~nm} \mathrm{~s}^{-1}$. Using eq (5), a grafted NP moiety rate of $13 \mathrm{pmol} \mathrm{cm}^{-2} \mathrm{~s}^{-1}$ is obtained taking for the grafted layer density ${ }^{29} \rho=1 \mathrm{~g} / \mathrm{mol}$ and for the molecular mass of the NP moiety $\mathrm{M}=122 \mathrm{~g} \mathrm{~mol}^{-1}$.

As demonstrated with other related coupled mass-deposition detection methods such as quartz-crystal microbalance $(\mathrm{QCM})^{29,30}$ or local ellipsometric imaging, ${ }^{11}$ the growth of the aryl multilayer is sustained by the flow of charge in the 
electrode and therefore the generation of the nitrophenyl (NP) radicals at the coated electrode. The good correlation between both the reflectivity variation and the charge, $\mathrm{Q}(\mathrm{t})$, for different times of the experiment shows that the overall grafting process requires the exchange of a constant amount of electrons per NBD molecule, $\mathrm{n}_{\mathrm{e}}$. The latter-can be estimated from eq (8) derived from Faraday's law:

$$
\mathrm{n}_{\mathrm{e}}=\frac{\mathrm{M}}{\mathrm{F} \rho \mathrm{A}} \frac{\mathrm{Q}(\mathrm{t})}{\operatorname{th}(\mathrm{t})}
$$

where $\mathrm{A}$ is the electrode surface area and $\mathrm{F}$ the Faraday's constant.

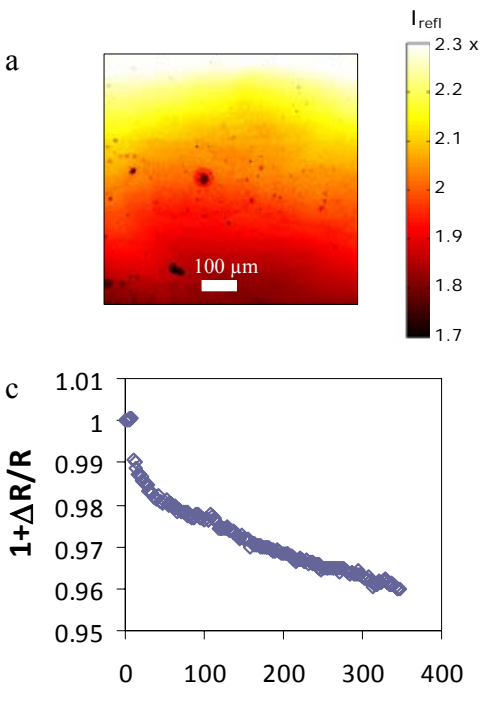

$\mathbf{t}(\mathbf{s})$
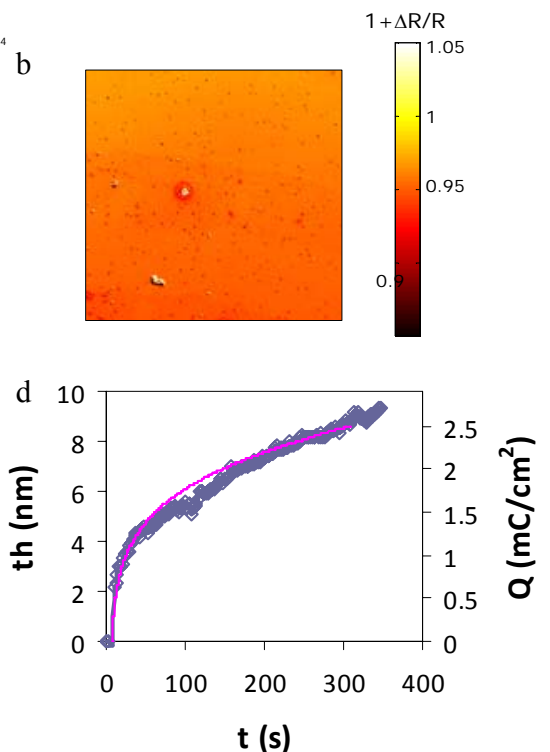

Fig. 3. Light reflectivity observation of NBD electrografting on an Au macroelectrode. a) image of the intensity of light reflected by the Au macroelectrode before the electrografting; b) image of the variation of light reflectivity at the end of the grafting (electrochemical actuation at $\mathrm{E}_{\mathrm{el}}=-0.4 \mathrm{~V}$ vs Ag/AgCl for $300 \mathrm{~s}$ ); c) evolution of the variation of light reflectivity (symbols) averaged on a $10 \times 10 \mu \mathrm{m}^{2}$ region during the grafting, transcribed d) into NP layer thickness by eq (4) and compared to the measured charge density (line).

An average value of $n_{e}=3.7$ is obtained for the macroscopic Au surface. If one assumes that one electron is required for the generation of one radical by the reductive cleavage of the diazonium ion, $n_{e}=3.7$ indicates that approximately three out of four generated radicals are not involved in the grafting process and are lost in competing chemical paths (e.g. radical reactions in solution). This is actually in agreement with diazonium electrografting processes monitored by other electrochemically-coupled techniques. ${ }^{11,29,30}$

\section{Vizualizing nitrophenyl radical reactions on microelectrode surfaces}

The versatility of light reflectivity microscopy relies on its simplicity of operation. Indeed, as for Au electrode surfaces, different electrode geometries and configurations can be monitored by this optical detection mode. Particularly, it can be applied to the monitoring of electrografting processes at microelectrodes that can be either microfabricated or made by standard glass-embedding techniques. Figure 4a presents the snapshots of the surface of a $25 \mu \mathrm{m}$ diameter $\mathrm{Au}$ microelectrode embedded in glass, respectively before the application of a constant $\mathrm{E}_{\mathrm{Au}}=-0.5 \mathrm{~V}$ vs $\mathrm{Ag} / \mathrm{AgCl}$ in a $5 \mathrm{mM} \mathrm{NBD}$ solution. The snapshot taken after $200 \mathrm{~s}$ of electrogragting and converted into a light reflectivity variation image is given in Figure 4b. As on macroscopic electrodes, the Au microelectrode electrografting is associated with an overall decrease of the Au surface reflectivity. Moreover, the heterogeneity of the reflected light intensity detected on the last image of the Au microelectrode grafting procedure (Figure $4 \mathrm{~b}$ ) is indicative of the heterogeneity of the thickness of the organic layer coating over the whole microelectrode. Even though these heterogeneities can be correlated to defects of the microelectrode (scratches), as was also detected on the macroscopic $\mathrm{Au}$ electrode in Figure $3 \mathrm{~b}$, it is also obvious that the edge of the microelectrode is particularly darker than its inner part. This qualitatively indicates the occurrence of higher grafting rates at electrode edges. 

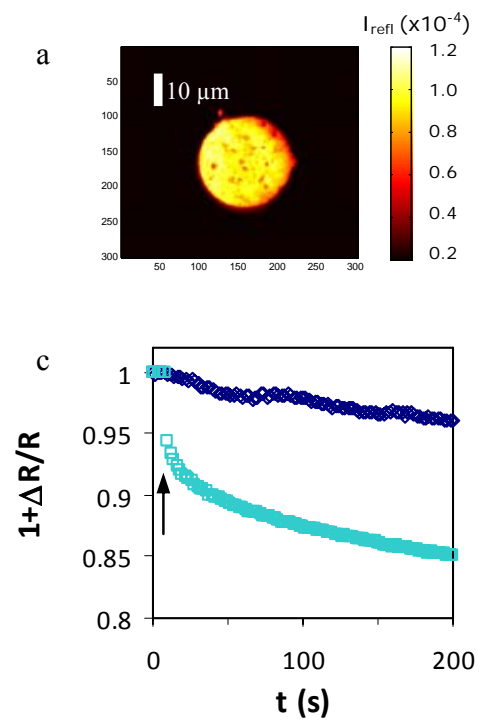

b
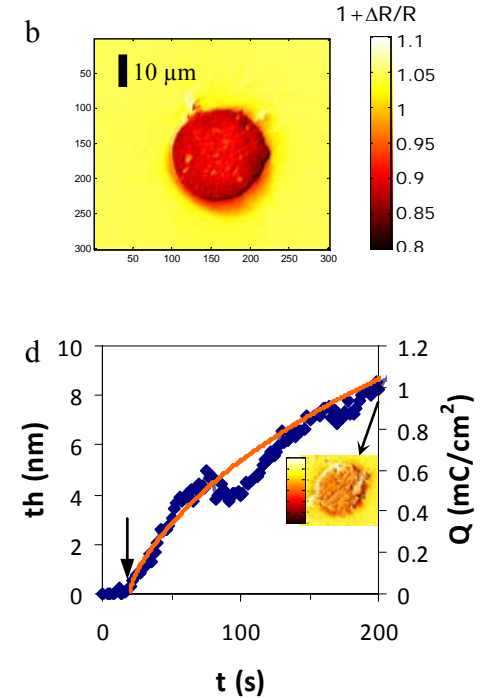

Fig. 4. Light reflectivity monitoring of the electrografting of NP layers on a $25 \mu \mathrm{m}$ diameter Au microelectrodes in the low flux regime. a) Light intensity image taken at $\mathrm{t}=0$ and b) light reflectivity image deduced from snapshot taken after $200 \mathrm{~s}$ of grafting actuated at $\mathrm{E}_{\mathrm{el}}=-0.5 \mathrm{~V}$ vs $\mathrm{Ag} / \mathrm{AgCl}$ in a $5 \mathrm{mM} \mathrm{NBD}+0.1 \mathrm{M} \mathrm{NBu}_{4} \mathrm{BF}_{4} \mathrm{ACN}$ solution. c) Change of light reflectivity averaged over $10 \times 10 \mu \mathrm{m}^{2} \mathrm{during}$ electrografting performed from top to bottom at $\mathrm{E}_{\mathrm{el}}=-0.3$ and $-0.5 \mathrm{~V}$ vs $\mathrm{Ag} / \mathrm{AgCl}$. d) $\mathrm{E}_{\mathrm{el}}=-0.3 \mathrm{~V}, \mathrm{NP}$ layer thickness variation (symbols) during the electrografting compared to measured charge (line); the inset is the light reflectivity image taken at $\mathrm{t}=200 \mathrm{~s}$; on the associated scale bar $1+\Delta \mathrm{R} / \mathrm{R}$ ranges from 1.02 to 0.9 . Vertical arrows indicate the initiation of the electrografting.

We have then evaluated quantitatively the heterogeneity of reaction rates on microelectrodes submitted to NBD electrografting. This is easily amenable by coupling the electrochemical initiation of the grafting at the microelectrode surface with its in situ and real time optical monitoring. We have then used light reflectivity imaging microscopy to monitor quantitatively the electrografting operated at different overall rates on Au microelectrodes. Indeed, the overall rate of the deposition of a thin NP layer can be tuned by adjustment of the microelectrode potential, $\mathrm{E}_{\mathrm{el}}$. Particularly in the case of the electroactive NP moieties, thick multilayers can be obtained when the electrografting is performed at $\mathrm{E}_{\mathrm{el}}$ close to $\mathrm{E}_{\mathrm{NP}}^{0}$, the standard reduction potential of nitrobenzene moieties. The NP moieties of the grafted layer then act as electron acceptor relays (through generation of the NP radical anions) to transport charges from the electrode to the NBD solution. A catalytic growth of NP multilayer is then observed. ${ }^{31,32}$ Under such condition, $\mathrm{E}_{\mathrm{el}} \sim \mathrm{E}_{\mathrm{NP}}^{0}$, the electrografting is performed in a regime of high driving force for the electrogeneration of NP radicals, while when $E_{\mathrm{el}}>>\mathrm{E}_{\mathrm{NP}}^{0}$ or $\mathrm{E}_{\mathrm{el}}$ close to $\mathrm{E}_{\mathrm{p}, \mathrm{NBD}}$, the peak potential associated to NBD reduction, the grafting is performed in a low driving force regime.

\section{A. Electrografting at low driving force $\left(\mathbf{E}_{\mathrm{NP}}^{0}<<\mathbf{E}_{\mathrm{el}} \sim \mathrm{E}_{\mathrm{p}, \mathrm{NBD}}\right)$}

In this regime, electrografting experiments have been performed at two different potentials, $\mathrm{E}=-0.3$ and $-0.5 \mathrm{~V}$ vs $\mathrm{Ag} / \mathrm{AgCl}$ and therefore at different overall rates. The overall rate can be evaluated from the time evolution of $1+\Delta \mathrm{R} / \mathrm{R}$ averaged over large domain $\left(>10 \times 10 \mu \mathrm{m}^{2}\right)$ of the microelectrode as presented in Figure 4c. The more negative the electrode potential, the more the reflectivity decreases (for th $<50 \mathrm{~nm}$ as seen in Figure $2 \mathrm{~b}$ ) and therefore the thicker the multilayer grows. The overall growth of the NP layer is also faster for both the induction period and the coarsening period when more negative $\mathrm{E}_{\mathrm{el}}$ is used. The experiment performed at $\mathrm{E}_{\mathrm{el}}=-0.5 \mathrm{~V}$ vs $\mathrm{Ag} / \mathrm{AgCl}$ informs on the reactivity of the electrogenerated NP radicals toward the NP multilayer and more generally can be used to assess the reactivity of the NP radical on surface-immobilised organic moieties.

The activation at $\mathrm{E}_{\mathrm{el}}=-0.3 \mathrm{~V}$ vs $\mathrm{Ag} / \mathrm{AgCl}$ presents a much slower grafting. The transcription of the reflectivity variation into organic layer thickness is shown in Figures $4 \mathrm{c}$ and $4 \mathrm{~d}$ and compared to the charge density variation detected at the microelectrode. As for the macroscopic electrode, the correlation between both quantities is good and an apparent number of electrons transferred per NBD molecule, $\mathrm{n}_{\mathrm{e}} \sim 1.6$, is obtained from eq (8) for the grafting of one NP radical on the Au microelectrode surface. During the early stage $(t<30 \mathrm{~s})$ of this grafting procedure, the monitoring of the NP monolayer formation reveals the reactivity of the NP radicals on the Au surface, which is characterized by an average layer growth rate of $v \sim 0.1 \mathrm{~nm} \mathrm{~s}^{-1}$ or an equivalent flux of reacting NP radicals of $80 \mathrm{pmol}$ $\mathrm{cm}^{-2} \mathrm{~s}^{-1}$. At longer times $(\mathrm{t}>30 \mathrm{~s})$, the layer growth is slower and ranges between $v=0.035$ to $0.06 \mathrm{~nm} \mathrm{~s}^{-1}$ (29 to 50 pmol $\mathrm{cm}^{-2} \mathrm{~s}^{-1}$ ).

The grafting at the $\mathrm{Au}$ microelectrode was operated in $\mathrm{E}_{\mathrm{el}}$ conditions comparable to the one presented on the 
macroscopic Au surface. Even though grafting rates on both surfaces are of the same order of magnitude, the NP layer deposition proceeds at a faster rate on the microelectrode than on the macroscopic Au surface.

We have next addressed the local grafting of the microelectrodes under both $\mathrm{E}_{\mathrm{el}}$ conditions. As shown in Figures $3 \mathrm{~b}$ and $4 \mathrm{~b}$, the coating thickness on the microelectrode at long grafting times is heterogeneous and reveals the heterogeneity of the grafting reactivity. This clearly shows the importance of surface defect sites and more importantly the higher coating along the edges of the microelectrode. The same fast grafting produced at $\mathrm{E}_{\mathrm{el}}=-0.5 \mathrm{~V}$, is analyzed at shorter times, during the induction period. Then, to reveal the heterogeneity of the grafting, Figures $5 \mathrm{a}, \mathrm{b}$ presents distribution images of the time derivative of local relative reflectivity changes, $d(\Delta R / R) / d t$, for $t=8$ and $12 \mathrm{~s}$ (the grafting starts at $t=10 \mathrm{~s}$ ). Even though data differentiation usually introduces noise, the microelectrode activity is clearly revealed as regions of $\mathrm{d}(\Delta \mathrm{R} / \mathrm{R}) / \mathrm{dt}<-0.02 \mathrm{~s}^{-1}$ or by layer thickness growth rates: $\mathrm{v}>4 \mathrm{~nm} \mathrm{~s}^{-1}$.

a
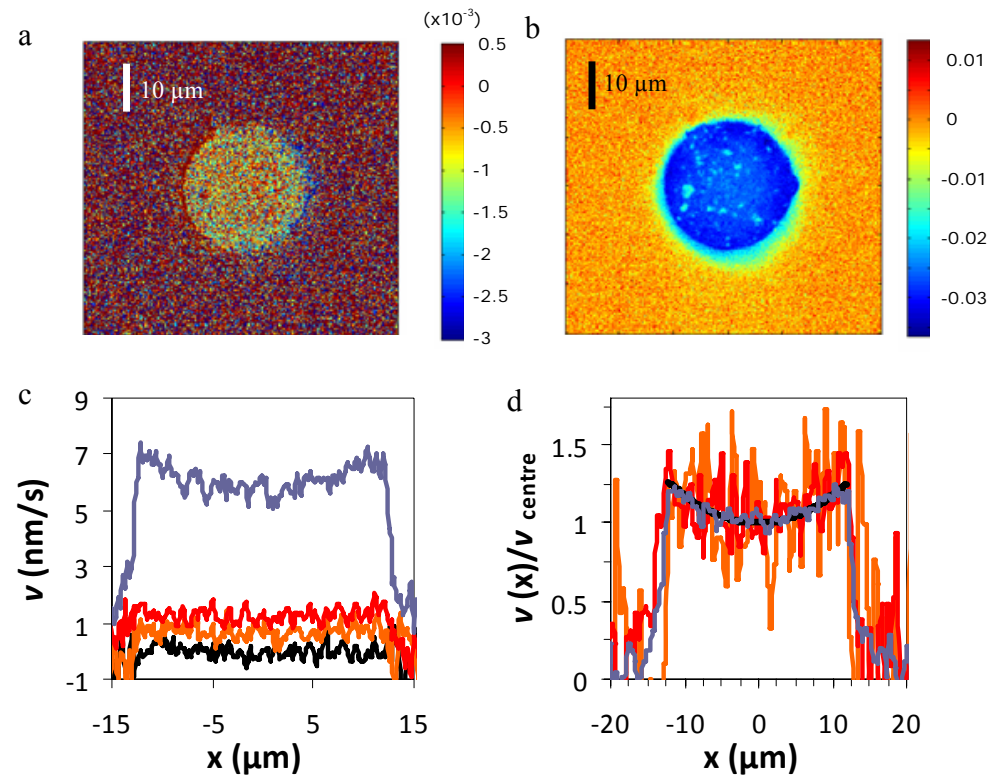

d

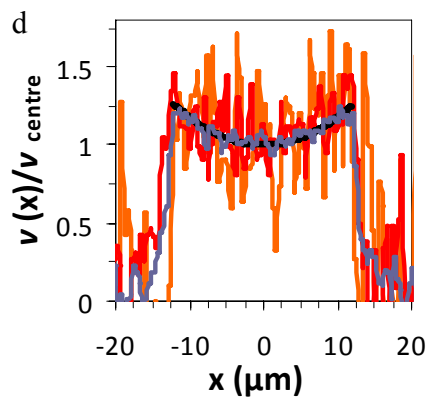

Fig. 5. Distribution of electrografting rates over a $25 \mu \mathrm{m}$ diameter Au microelectrode, $\mathrm{E}_{\mathrm{el}}=-0.5 \mathrm{~V}$ vs $\mathrm{Ag} / \mathrm{AgCl}$. a,b) Image of reflectivity variation $\mathrm{d}(\Delta \mathrm{R} / \mathrm{R}) / \mathrm{dt}$ at 8 (a) and $12 \mathrm{~s}$ (b). The color bars correspond to values of $\mathrm{d}(\Delta \mathrm{R} / \mathrm{R}) / \mathrm{dt}^{\mathrm{in} \mathrm{s}} \mathrm{s}^{-1}$. c, d) Distribution of the grafting rate (c) over a microelectrode diameter at $\mathrm{t}=12,14,16,8 \mathrm{~s}$ (from top to bottom) or (d) normalized by the rate at the microelectrode center (black line: simulated distribution rate with $\left.\mathrm{k}=510^{-4} \mathrm{~cm} \mathrm{~s}^{-1} \mathrm{k}_{\mathrm{c}}>100 \mathrm{~s}^{-1}\right)$.

This image also reveals heterogeneous regions as lighter micrometric areas of slower coating rates. Except from these individual defects, a cross section of the growth rate, $v(\mathrm{x})$, along a microelectrode diameter is presented in Figure $5 \mathrm{c}$ for $\mathrm{t}=8,12,14$ and $16 \mathrm{~s}$. For $\mathrm{t}>10 \mathrm{~s}$, spatio-temporal variations of the reactivity of NP radicals on the surface are clearly seen. Indeed, the grafting rate $v(\mathrm{x})$ depends on the radial position on the microelectrode. As observed at longer times in Figure $4 b$, microelectrode regions closer to the edge are regions of faster NP radicals grafting. The ratio of edge-to-centre grafting rates is however rather small $v_{\text {edge }} / v_{\text {centre }}=1.27$. As observed for $\mathrm{t}=14$ and $16 \mathrm{~s}$, the grafting rate greatly decreases with time, but the radial evolution of the grafting rate is still observed. At longer times $t>30 \mathrm{~s}$ the layer growth stabilizes to a constant rate of $0.09 \mathrm{~nm} \mathrm{~s}^{-1}\left(74 \mathrm{pmol} \mathrm{cm}^{-2} \mathrm{~s}^{-1}\right)$ with still an apparent lower reflectivity around the edge of the microelectrode than on its centre (Figure 4b). Even if this experiment actually characterizes the reactivity on an already coated surface and therefore on surface-immobilised NP moieties, it suggests that the spatiotemporal reactivity of the NP radical is maintained along the whole grafting process.

Similar analysis during the early stage of $\mathrm{Au}$ microelectrode monolayer coverage is more delicate as the signal detected when $\mathrm{E}_{\mathrm{el}}=-0.3 \mathrm{~V}$ is close to the limit of detection of the optical method $(\sim 0.002$ in term of $1+\Delta \mathrm{R} / \mathrm{R})$. However, the grafting rate is not significantly different along the different positions of the microelectrode, and again the $v_{\text {edge }} / v_{\text {centre }}$ ratio is likely close to 1 .

\section{B. Electrografting at high driving force $\left(\mathrm{E}_{\mathrm{NP}}^{0} \sim \mathrm{E}_{\mathrm{el}}<<\mathrm{E}_{\mathrm{p}, \mathrm{NBD}}\right)$}

A similar analysis has been undertaken at much higher NP radical flux regimes. This is obtained by performing the electrografting at $\mathrm{E}_{\mathrm{el}}$ where the NP radical anion can be formed (typically $\mathrm{E}_{\mathrm{el}}==-0.9 \mathrm{~V} \mathrm{vs} \mathrm{Ag} / \mathrm{AgCl}$ ). Here, thick NP multilayers are obtained and their generation is sustained by the NP immobilized units. The latter act as redox catalytic relays for electron transfer to propagate electrons from the electrode to the NBD solution for further NP radical generation. A typical example of reflectivity variation during such thick NP multilayer generation is presented in Figure $6 \mathrm{a}$ and compared to the evolution of the charge transferred electrochemically for the NBD reduction. 
The reflectivity variations clearly show oscillations, as predicted optically (see eq. (5)), even though the average variation decreases and deviates from the theoretical prediction for a transparent layer. It is not the aim of this work to explain the origin of such deviation and this will be described elsewhere. Even without theoretical agreement, approximate layer thicknesses may be obtained at the maximum and minimum of $1+\Delta R / R$. These characteristic occurrences should be met for destructive or constructive interferences and therefore they correspond to layer thicknesses similar to those observed in Figure $2 \mathrm{~b}$. For an illumination at $\lambda=490 \mathrm{~nm}$ and a layer of refractive index $\mathrm{n}_{\mathrm{L}}$ $=1.5$, the first minimum corresponds to the deposition of a $51 \mathrm{~nm}$ thick layer; and the layer grows by $\Delta$ th $\sim \lambda / 4 \mathrm{n}_{\mathrm{L}}=82$ $\mathrm{nm}$ between two consecutive minimum and maximum. As observed in Figure $6 \mathrm{a}$, the grafting performed a $\mathrm{E}_{\mathrm{el}}=-0.9 \mathrm{~V}$ vs $\mathrm{Ag} / \mathrm{AgCl}$ yields a multilayer of thickness th $>135 \mathrm{~nm}$, in agreement with the possible generation of very thick multilayers ${ }^{30,31}$ under such high driving force regime for NP radical generation.

Owing to the coarse optical model used here, it is difficult to correlate the light reflectivity change to the electrochemical charge transferred during the whole experiment. However, for an optical observation made at the microelectrode centre (red symbols in Figure $6 \mathrm{a}$ ), the extrema of $1+\Delta \mathrm{R} / \mathrm{R}$ correspond to a constant layer growth rate (red symbols in Figure $6 \mathrm{~b}$ ) of $v=0.36 \mathrm{~nm} \mathrm{~s}^{-1}$ or equivalently $300 \mathrm{pmol} \mathrm{cm}^{-2} \mathrm{~s}^{-1}$. Such growth rate is about 4 to 6 times faster than that observed on microelectrodes at lower driving force $\left(\mathrm{E}_{\mathrm{el}}=-0.5\right.$ and $-0.3 \mathrm{~V}$ respectively). This shows the impact of the driving force on the generation of the NP radicals, that is the impact of the electrode potential, $\mathrm{E}_{\mathrm{el}}$, on the grafting rate. Moreover, as observed for NP grafting at lower driving force, the constant $v$ at long time is also consistent with the linear variation of the charge with the NBD reduction time by $0.035 \mathrm{mC} \mathrm{cm}^{-2} \mathrm{~s}^{-1}$. From eq (8), an average number of electrons $\mathrm{n}_{\mathrm{e}} \sim 1.23$ is transferred per NBD molecule on this $250 \mu \mathrm{m}$ diameter Au electrode.

The characteristic reflectivity variation also allows the interpretation of the spatio-temporal distribution of the grafting on the electrode in these very fast coating regimes. Figure 6 a presents the time evolution of the reflectivity changes estimated at different locations on the $250 \mu \mathrm{m}$ radius electrode, as indicated by the arrow in Figure 6a and the positions represented in Figure $6 \mathrm{~b}$. The spatial distribution of the grafting is deduced from the apparent arrival of the reflectivity wavefront at the different positions on the electrode. Clearly, the wavefront first reaches the edge of the electrode. It propagates and is delayed when going to its centre. Quantitative spatio-temporal analysis is obtained from the estimate of the grafting rate at the time of arrival of the minimum and maximum reflectivity along the different positions on the microelectrode. The variations of this grafting rate are presented in Figure $6 \mathrm{~b}$. Again, faster rates are observed in the microelectrode edge region than at its centre, with an edge-to-centre ratio $v_{\text {edge }} / v_{\text {centre }}=1.40 \pm 0.04$, closely related to that observed at lower NP driving force.
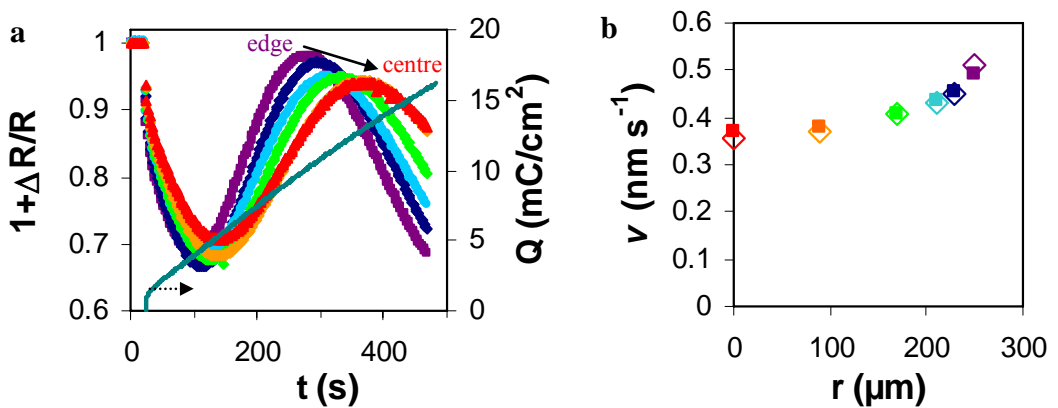

Fig. 6. Growth of a NP multilayer of thickness th $>130 \mathrm{~nm}$ on a $500 \mu \mathrm{m}$ diameter disk Au electrode $\left(\mathrm{E}_{\mathrm{el}}=-0.9 \mathrm{~V} \mathrm{vs} \mathrm{Ag} / \mathrm{AgCl}\right)$. a) $\mathrm{Light}$ reflectivity measured at different locations along a radius of the electrode going from the edge to the centre, compared to electrochemical charge density variation (grey-blue line). b) Evolution along the electrode radius $(r=0$ : centre, $r=250 \mu \mathrm{m}$ : edge) of the local grafting rate estimated assuming resp. $51(\mathbf{\square})$ and $133(\diamond) \mathrm{nm}$ are grafted at the minimum and maximum of $1+\Delta \mathrm{R} / \mathrm{R}$.

\section{Tentative mechanistic interpretation}

\section{Summary of the results}

The electrochemical actuation of the NBD reductive electrografting allows one to tune the overall grafting of NP layers on $\mathrm{Au}$ electrode surfaces with growth rates, $v$, spanning over $\sim 2$ orders of magnitude: from $0.035 \mathrm{~nm} \mathrm{~s}^{-1}$ (long time and low driving force $\mathrm{E}_{\mathrm{el}}=-0.3 \mathrm{~V}$ ) to $0.36 \mathrm{~nm} \mathrm{~s}^{-1}$ (long time and high driving force $\mathrm{E}_{\mathrm{el}}=-0.9 \mathrm{~V} \mathrm{vs} \mathrm{Ag} / \mathrm{AgCl}$ ) to $>$ $2 \mathrm{~nm} \mathrm{~s}^{-1}$ during the fast induction period (short time $\mathrm{E}_{\mathrm{el}}=-0.5 \mathrm{~V}$ ). Coupling such electrochemical actuation of thin NP organic layer coatings on a reflecting microelectrode surface to its optical detection then allows one to monitor the local grafting rates or the local fluxes of reacting NP radicals. It provides an indirect detection of the local electrochemical current densities associated with the NBD reduction.

Over the whole range of fluxes/growth rates investigated, the NP layer deposition rate is slightly higher at the microelectrode edges (and defects) than at its centre. At first sight, this is in line with edge effects such as the generation of higher diffusion fluxes of electrogenerated reactive species at the edge of a microelectrode rather than at its centre. If this has been theoretically predicted, it has scarcely been demonstrated experimentally. ${ }^{17,19-22,33} \mathrm{We}$ 
believe that the observed grafting rate distribution provides another qualitative illustration of the manifestation of such edge effects.

\section{Simplified kinetic model for NBD electrografting}

To get a more quantitative insight into the phenomenon, the electrochemical process leading to the NP layer deposition should be modelled. Even though the electrografting of surfaces by reduction of aryldiazonium salts is largely employed and different mechanistic aspects have been discussed, ${ }^{34-36}$ a mechanism that encompasses satisfactorily the whole aspects of the grafting has not been proposed yet. This objective is particularly difficult to reach as the aryldiazonium reduction yields reactive species that are prone to react with a wide range of materials, such as their generating electrode surface but also the deposited film or the close environment of the electrode. Moreover, if the reaction of the electrogenerated radical with the surface leads to the modification (partial attenuation) of its electrochemical properties, it hardly blocks it completely allowing for a continuous radical generation and sustained grafting process. It is therefore not reasonable to propose a mechanism based on these results, and we will only concentrate on a tentative interpretation of the observed phenomenon following a simplified approach proposed by Savéant. ${ }^{37}$

The grafting of a surface by aryldiazonium salts, $\mathrm{ArN}_{2}{ }^{+}$, results from the initial dediazoniation of the aryldiazonium leading to the generation of a reactive species. The mechanism of such preliminary step and the nature of the reactive step is controversy. Depending on the experimental conditions and mostly of the solvent, actuation and temperature either heterolytic or homolytic dediazoniation routes have been proposed and more or less recently reviewed. ${ }^{38-40}$ As described by Zollinger, ${ }^{38,41}$ the heterolytic dediazoniation (9) yields a reactive aryl cation, $\mathrm{Ar}^{+}$, through intermediate caged fragments.

$$
\mathrm{ArN}_{2}^{+} \square \quad\left[\mathrm{Ar}^{+}, \mathrm{N}_{2}\right] \square \quad \mathrm{Ar}^{+}+\mathrm{N}_{2}
$$

Such $\mathrm{S}_{\mathrm{N}} 1$ mechanism and carbocation intermediate can be observed in solvents of low nucleophilicity such as water or acetonitrile and is favoured at elevated temperature. ${ }^{42}$ Further reduction of the aryl carbocation into an aryl radical maybe invoked owing to its highly positive potential. However the characteristic time for the aryl carbocation generation is of the order of several hours, $>3$ orders of magnitude higher than that imposed by the electrochemical activation proposed here.

Homolytic dediazoniation prevails in more nucleophilic solvents or conditions ${ }^{43}$ and more particularly when electron is transferred to the aryldiazonium salt as in the case of electrografting processes. In the following we consider, as generally admitted, ${ }^{44}$ that NP radicals, denoted $\mathrm{Ar}^{\circ}$, are generated from the irreversible electrochemical reduction of NBD according to a concerted electron transfer bond-breaking process following Savéant's denomination: ${ }^{45}$

$$
\mathrm{NBD}+\mathrm{e} \stackrel{\mathrm{k}}{\longrightarrow} \mathrm{Ar}^{\bullet}+\mathrm{N}_{2}
$$

The NBD consumption at the electrode is described either by mass-transfer limitation or by kinetic limitation from the charge transfer process associated to the rate constant $\mathrm{k}$ (in $\mathrm{cm} \mathrm{s}^{-1}$ ). The flux of consumed NBD is then given, on the one hand, from a Butler-Volmer-like relationship in the case of kinetic limitation:

$$
\left.\mathrm{D} \frac{\partial[\mathrm{NBD}]}{\partial \mathrm{z}}\right|_{\mathrm{z}=0}=\mathrm{k}[\mathrm{NBD}]_{\mathrm{z}=0}
$$

where $\mathrm{z}$ is the direction normal to the electrode surface, $[\mathrm{NBD}]_{\mathrm{z}=0}$ the concentration of NBD at the electrode surface and D the NBD diffusion coefficient. On the other hand, for fast charge transfer, the boundary condition at the electrode surface, eq (12), implies that the concentration of NBD at the electrode surface, $[N B D]_{z=0}$ is constant for any radial position, $r \leq \mathrm{a}$ (a: electrode radius), and its value is dictated by the electrode potential:

$$
[\mathrm{NBD}]_{\mathrm{z}=0, \mathrm{r} \leq \mathrm{a}}=\mathrm{cst}
$$

The flux of NBD is then obtained from the resolution of the diffusion equation, eq (13), in both directions: radial, $r$, and normal, $z$, to the electrode surface.

$$
\frac{\partial[\mathrm{NBD}]}{\partial \mathrm{t}}=\mathrm{D}\left(\frac{\partial^{2}[\mathrm{NBD}]}{\partial \mathrm{r}^{2}}+\frac{1}{\mathrm{r}} \frac{\partial[\mathrm{NBD}]}{\partial \mathrm{r}}+\frac{\partial^{2}[\mathrm{NBD}]}{\partial \mathrm{z}^{2}}\right)
$$

The flux of NBD consumption is also the rate of production of NP radicals, which can diffuse away in solution from their generating electrode surface and also react on the electrode surface to grow the NP layer. Comparison to the experimental observation indicates that at long times, both the overall grafting rate and the electrochemical current are constant (steady increase of thickness and charge). It then allows a rough estimate of the average flux of NBD consumption at the electrode surface: 


$$
\left.\mathrm{D} \frac{\mathrm{d}[\mathrm{NBD}]}{\mathrm{dz}}\right|_{\mathrm{z}=0}=\frac{\mathrm{i}}{\mathrm{FS}} \approx \frac{\Delta \mathrm{Q}}{\mathrm{FS} \Delta \mathrm{t}}
$$

From the long time behaviours observed in Figures 3, 4 and 6, this flux is $\sim 10^{-10} \mathrm{~mol} \mathrm{~cm}^{-2} \mathrm{~s}^{-1}$. This is considerably small compared to the steady-state flux that can be sustained by a $25 \mu \mathrm{m}$ diameter microelectrode for complete reduction of a $5 \mathrm{mM} \mathrm{NBD}$ solution $\left(4 \mathrm{DC}^{0} /(\pi \mathrm{a})=5 \times 10^{-8} \mathrm{~mol} \mathrm{~cm}^{-2} \mathrm{~s}^{-1}\right)$. It suggests that the long time grafting is performed under slow charge transfer rate for the NBD reduction (boundary (11) holds instead of (12)), and that the NBD concentration at the electrode is close tot the bulk concentration, $[\mathrm{NBD}]^{0}$. An estimate of the charge transfer rate, $\mathrm{k}$, is then given by:

$$
\frac{\Delta \mathrm{Q}}{\mathrm{FS} \Delta \mathrm{t}} \approx \mathrm{k}[\mathrm{NBD}]^{0}
$$

which yields a lower-bound of the apparent NBD consumption rate of $\mathrm{k} \sim 2 \times 10^{-5} \mathrm{~cm} \mathrm{~s}^{-1}$ considering that the whole electrode surface is active.

In a simplified model, the NP radical grafting on the electrode surface assumes that NP radicals react with any part of the surface (bare Au surface or NP multilayer):

$$
\mathrm{Ar}^{\bullet}+\text { Surface } \stackrel{\mathrm{k}_{\mathrm{gr}}}{\longrightarrow} \text { Surface-NP }
$$

The evolution of $\Gamma$, the surface concentration of the grafted NP, Surface-NP in eq (16), is then described by a firstorder law (eq (17)) respective to the concentration of the reactive NP radical, Ar ${ }^{\circ}$, at the solution/electrode interface:

$$
\frac{\mathrm{d} \Gamma}{\mathrm{dt}}=\mathrm{k}_{\mathrm{gr}}\left[\mathrm{Ar}^{\bullet}\right]_{\mathrm{z}=0}
$$

In eq (17), $\mathrm{k}_{\mathrm{gr}}$ characterizes the grafting reaction rate constant (in $\mathrm{cm} \mathrm{s}^{-1}$ ), which contains the overall diversity of the grafting process. For example, it may include the difference of reaction rates of the NP radical with the bare electrode surface or with the covering NP multilayer. It may also include the number of active sites of the surface, or their surface concentration, which are prone to be grafted and to sustain the layer growth. In a simplified version, we assume the reaction rate $\mathrm{k}_{\mathrm{gr}}$ is constant over the whole grafting process.

Eqs (10) and (16) indicate that the rate of production of $\mathrm{Ar}^{\bullet}$ is balanced by two competing routes from the electrode surface: their escape by diffusion and their grafting. This gives :

$$
-\left.\mathrm{D} \frac{\partial[\mathrm{Ar} \cdot]}{\partial \mathrm{z}}\right|_{\mathrm{z}=0}=\mathrm{k}[\mathrm{NBD}]_{\mathrm{z}=0}-\mathrm{k}_{\mathrm{gr}}\left[\mathrm{Ar}^{\bullet}\right]_{\mathrm{z}=0}
$$

Based on eq (17), the grafting rate $v=\mathrm{d} \Gamma / \mathrm{dt}$ and also the amount of grafted material, $\Gamma$, should reflect the concentration profile of the NP radical, $\mathrm{Ar}^{\bullet}$, above the electrogenerating electrode surface. The problem then relies on the resolution of the diffusion equations for NBD and $\mathrm{Ar}^{\bullet}$ (eq (13)), taking into account the boundary conditions given by (11) and (18).

\section{Simulated distribution of NP radical over the electrode surface}

As observed experimentally for long grafting rates, the NBD consumption (or Ar generation) at the electrode is slow compared to the radical attack on the surface $\left(\mathrm{k}<<\mathrm{k}_{\mathrm{gr}}\right)$; this is consistent with the observed values of $\mathrm{k}_{\mathrm{gr}}$ of the order of $1 \mathrm{~cm} \mathrm{~s}^{-1}$ for the growth of polyphenyl multilayers. ${ }^{36}$ The electrode coating is then dictated by the generation/diffusion of the NP radical from the electrode and the electrode coating follows the diffusion-controlled concentration profile of $\mathrm{Ar}^{\bullet}$ above the electrode surface acting as a source of NP radicals. These concentration profiles $\left[\mathrm{Ar}^{\bullet}\right]_{\mathrm{z}=0}$ at the

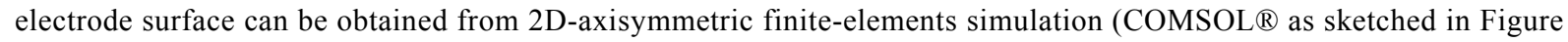
7a). An example is shown in Figures $7 \mathrm{a}$ and $7 \mathrm{~b}$ that present respectively the spatial distribution of the NBD concentration in solution or the evolution of $\mathrm{Ar}^{\bullet}$ concentration along the radial coordinate, $\mathrm{r}$, generated above a source electrode, of radius $\mathrm{a}=250 \mu \mathrm{m}$ (mimicking the experiment of Figure 6$)$, using $\mathrm{k}=2 \times 10^{-5} \mathrm{~cm} \mathrm{~s}^{-1}$ and two extreme values of the grafting rate $\mathrm{k}_{\mathrm{gr}}$ of $2 \times 10^{-3}$ (Figures $7 \mathrm{a}$ and $7 \mathrm{~b}$ ) and $2 \mathrm{~cm} \mathrm{~s}^{-1}$ (Figure $7 \mathrm{~b}$ ) respectively.

Both situations yield a flux of diffusing $\mathrm{Ar}^{*}$, which diverges at the electrode edge (not shown), and this illustrates the known electrode edge effect. Obviously from examination of the boundaries (11) and (12) for $\mathrm{Ar}^{\bullet}$ generation at the electrode, only the kinetically-limited charge transfer situation can introduce some radial evolution of the electrogenerated $\mathrm{Ar}^{\circ}$ radical and therefore some spatial evolution of the grafting rate along the electrode. This is indeed confirmed from the simulated concentration profiles of $\mathrm{Ar}^{\bullet}$ given in Figure $7 \mathrm{~b}$. However, the charge transfer kinetic limitation at the microelectrode rather predicts higher [ $\left.\mathrm{Ar}^{\bullet}\right]$ and therefore a higher grafting rate at the microelectrode centre than at the edges. Increasing $\mathrm{k}_{\mathrm{gr}}$ to values $>0.2 \mathrm{~cm} \mathrm{~s}^{-1}$ allows to inverse the Ar spatial distribution over the electrode surface but the concentration is only $1 \%$ higher at the electrode edges than at its centre. For both limiting $\mathrm{k}_{\mathrm{gr}}$ values, the predicted grafting distributions are in disagreement with the experimental 
observations.

The experimentally observed inverted grafting distribution then suggests, based on the simple grafting model proposed, that higher $\mathrm{Ar}^{\bullet}$ concentrations are found at the microelectrode edge while they are also regions of higher NBD concentrations. This can be met if considering that either (i) NBD and/or Ar have different intrinsic reactivities at the electrode centre vs edge or more simply if (ii) $\mathrm{Ar}^{\bullet}$ presents some chemical instability in the solution phase.

\section{Influence of the NP radical chemical stability}

As a tentative first hint, we consider the simplest second possibility as phenyl radicals are indeed highly reactive species and are scavenged in solution at a rate $\mathrm{k}_{\mathrm{c}}$ according to:

$$
\mathrm{Ar}^{\bullet} \stackrel{\mathrm{k}_{\mathrm{c}}}{\longrightarrow} \text { product }
$$

which is accounted by the introduction in the right-hand part of the diffusion equation of $\mathrm{Ar}^{\bullet}$ (eq 13 with $\mathrm{Ar}^{\bullet}$ instead of NBD) of a consumption term $-\mathrm{k}_{\mathrm{c}}\left[\mathrm{Ar}^{\bullet}\right.$ ]. If the chemical scavenging of $\mathrm{Ar}^{\bullet}$, the NP radical, is likely not the only regulating matter, it is also a reasonable explanation for the low electrochemical efficiency of the grafting $\left(\mathrm{n}_{\mathrm{e}}>1\right)$. In view of this simple model, the grafting efficiency is obtained from comparison of both competing routes for $\mathrm{Ar}^{\bullet}$ consumption: its grafting on the electrode at a rate $\mathrm{k}_{\mathrm{gr}} \mathrm{vs}$ its solution diffusion/scavenging at a rate $\left(\mathrm{Dk}_{\mathrm{c}}\right)^{1 / 2}$. The apparent number of exchanged electron is then given by: ${ }^{37,46,47}$

$$
\frac{1}{\mathrm{n}_{\mathrm{e}}}=\frac{\mathrm{k}_{\mathrm{gr}}}{\mathrm{k}_{\mathrm{gr}}+\sqrt{\mathrm{Dk}_{\mathrm{c}}}}
$$

Indeed, phenyl radicals are known to be scavenged in various radical reactions and particularly via $\mathrm{H}$-atom abstraction in most common organic solvents. This reaction has actually a reaction rate constant estimated as $\mathrm{k}_{\mathrm{c}}>10^{6}$ $\mathrm{s}^{-1}$ in $\mathrm{ACN},{ }^{48}$ that is fast enough to impose the heterogeneity of [ $\left.\mathrm{Ar}^{-}\right]$above the eletrogenerating electrode surface. For a value of the diffusion coefficient of $10^{-5} \mathrm{~cm}^{2} \mathrm{~s}^{-1}$, the observed values of $\mathrm{n}_{\mathrm{e}}$ (from 1.2 to 4 ) ensure values of $\left(\mathrm{Dk}_{\mathrm{c}}\right)^{1 / 2} / \mathrm{k}_{\mathrm{gr}}$ of the order of 0.2 to 3 , yielding values of $\mathrm{k}_{\mathrm{gr}}$ in the 1 to $15 \mathrm{~cm} \mathrm{~s}^{-1}$ range, in agreement with values described for the growth of polyphenyl multilayers. ${ }^{36}$

This homogeneous scavenging can be implemented ${ }^{13,49,50}$ in the problem of diffusion/solution reaction/grafting of $\mathrm{Ar}^{\bullet}$ as presented in Figure $7 \mathrm{~b}$ when considering as negligible the contribution from the grafting reaction, $\mathrm{k}_{\mathrm{gr}}=2 \times 10^{-3}$ $\mathrm{cm} \mathrm{s}^{-1}$, and observing the effect of $\mathrm{k}_{\mathrm{c}}=10^{3} \mathrm{~s}^{-1}$. As a fast grafting rate, the solution scavenging of Ar results in the observation of a higher [ $\left.\mathrm{Ar}^{*}\right]$ at the electrode edges than at its centre and the same limiting concentration profile is observed ( $1 \%$ higher concentration at the electrode edges).

Therefore, whatever the value of the $\mathrm{Ar}^{\bullet}$ generation rate $\mathrm{k}$, adjusting its surface grafting rate, $\mathrm{k}_{\mathrm{gr}}$, and/or its homogeneous scavenging rate, $\mathrm{k}_{\mathrm{c}}$, allows one to obtain a limiting situation for which the edges of the electrode become regions of higher [ $\left.\mathrm{Ar}^{\bullet}\right]$. The radial distribution of the simulated grafting rate in the limited situation is then related to the value of $\mathrm{k}$ (the higher $\mathrm{k}$, the higher the possible the edge effect). To reproduce satisfactorily the experimentally observed evolution at both the 500 and $25 \mu \mathrm{m}$ diameter electrodes, a minimum value of $\mathrm{k}=0.001$ and $510^{-4} \mathrm{~cm} \mathrm{~s}^{-1}$ respectively is necessary as shown in Figure $7 \mathrm{c}$ for the $500 \mu \mathrm{m}$ diameter electrode and in Figure 5d (black line) for the $25 \mu \mathrm{m}$ one. Despite its crudeness, the proposed mechanism predicts a distribution of grafting rates (higher rate at the electrode edge) in good agreement with those detected experimentally. It is not reasonable to provide a deeper analysis (determination of $\mathrm{k}_{\mathrm{gr}}, \mathrm{k}$ and $\mathrm{k}_{\mathrm{c}}$ ) or a more complex mechanistic approach as it requires too many unknown parameters.
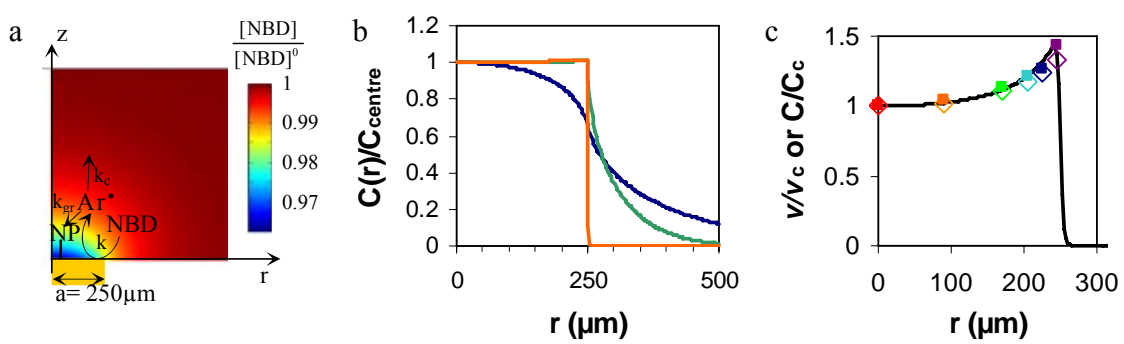

Fig. 7 Simulation of NP radical, $\mathrm{Ar}^{\bullet}$, generation at a $250 \mu \mathrm{m}$ radius electrode mimicking the experimental grafting of Figure 6 . a) Schematic description of the problem; b) simulated normalized concentration profile of $\left[\mathrm{Ar}^{\bullet}\right]_{\mathrm{z}=0}$ at $100 \mathrm{~s}$ along the electrode radial coordinate, $\mathrm{r}$, for kinetically-limited situations for $\mathrm{k}=2 \times 10^{-5} \mathrm{~cm} \mathrm{~s}^{-1}$ : low-consumption regime (blue line), $\mathrm{k}_{\mathrm{c}}=0, \mathrm{k}_{\mathrm{gr}}=2 \times 10^{-3} \mathrm{~cm} \mathrm{~s}^{-1}$, high-consumption regime for (green) $\mathrm{k}_{\mathrm{c}}=0$ and $\mathrm{k}_{\mathrm{gr}}=2 \mathrm{~cm} \mathrm{~s}^{-1}$ or (orange) $\mathrm{k}_{\mathrm{c}}>10^{2} \mathrm{~s}^{-1}$ and $\mathrm{k}_{\mathrm{gr}}=2 \times 10^{-3} \mathrm{~cm} \mathrm{~s}^{-1}$; c) comparison of the simulated concentration profile for k= $10^{-3} \mathrm{~cm} \mathrm{~s}^{-1}, \mathrm{k}_{\mathrm{gr}}=2 \times 10^{-3} \mathrm{~cm} \mathrm{~s}^{-1} \mathrm{k}_{\mathrm{c}}>10^{2} \mathrm{~s}^{-1}$ with the experimental radial distribution of the grafting rate (from Figure $6 \mathrm{~d}$ ). 


\section{Conclusion}

The coating of gold ( $\mathrm{Au}$ ) electrode surfaces with nitrophenyl (NP) layers was studied by combination of electrochemical actuation and optical detection of the grafting process. The electrochemical actuation of the reduction of the nitrobenzenediazonium (NBD) precursor is used to generate NP radicals and therefore initiate the electrografting. This electrografting process is followed in situ and in real time by light reflectivity microscopy imaging, allowing for spatio-temporal observation with sub-micrometric lateral resolution and sub-nm thickness sensitivity in the case of a reflecting Au electrode coated by a transparent organic layer. The interest of an electrochemical actuation is its ability to finely control the electrode potential and therefore to tune the overall grafting of NP layers onto Au electrode surfaces with growth rates, $v$ spanning over almost 2 orders of magnitude: from 0.035 $\mathrm{nm} / \mathrm{s}$ (long time and low driving force, $\mathrm{E}_{\mathrm{el}}=-0.3 \mathrm{~V} \mathrm{vs} \mathrm{Ag} / \mathrm{AgCl}$ ) to $0.36 \mathrm{~nm} / \mathrm{s}$ (long time and high driving force, $\mathrm{E}_{\mathrm{el}}=$ $0.9 \mathrm{~V}$ vs $\mathrm{Ag} / \mathrm{AgCl}$ ) to $>2 \mathrm{~nm} / \mathrm{s}$ during the fast induction period (short time $\mathrm{E}_{\mathrm{el}}=-0.5 \mathrm{~V} \mathrm{vs} \mathrm{Ag} / \mathrm{AgCl}$ ). Coupling such an electrochemical actuation of a thin NP organic layer coating on a reflecting microelectrode surface to its optical detection then allowed monitoring the local grafting rates or the local fluxes of reacting NP radicals.

Over the whole range of fluxes/growth rates investigated, the NP layer deposition rate is slightly higher at the microelectrode edges (and defects) than at its centre. At first sight, this is in line with edge effects such as the generation of higher diffusion fluxes of electrogenerated reactive species at edges of a microelectrode rather than at its centre. If this has been theoretically predicted, it has been scarcely demonstrated experimentally. We believe that the observed grafting rate distribution provides another qualitative illustration of the manifestation of such edge effect phenomenon. Despite the general complexity of the mechanism involved in diazonium electrografting processes, a simplified model showed how the chemical instability of the electrogenerated radical (by fast scavenging in solution or fast grafting at the electrode) is a possible source of the observed distribution of grafting rates over the electrode surface.

More generally, this work demonstrates the potentiality of combined opto-electrochemical microscopy to image surface reaction kinetics from the quantification (or indirect detection) of the local electrochemical current densities associated with these surface reactions. This is demonstrated here in the case of NP layer grafting from NBD reduction and it can be generalized to a wide range of surface transformation processes.

Acknowledgments. The "Agence Nationale de la Recherche", ANR, is gratefully acknowledged for its financial support via the ANR-08-JCJC0088 $\mu$ ECOLIERS project.

\section{References}

B. M. Weckhuysen, Angew. Chem. Intl. Ed., 2009, 48, 4910.

2 X. Zhou, N. M. Andoy, G. Liu, E. Choudhary, K.-S. Han, H. Shen, P. Chen, Nat Nano, 2012, 7, 237.

3 A. Meunier, O. Jouannot, R. Fulcrand, I. Fanget, M. Bretou, E. Karatekin, S. arbault, M. Guille, F. Darchen, F. Lemaître, C. Amatore, Angew. Chem. Intl. Ed., 2011, 50, 5081.

4 C. Amatore, C. Pebay, L. Servant, N. Sojic, S. Szunerits, L. Thouin, ChemPhysChem, 2006, 7, 1322.

5 C. Amatore, F. Bonhomme, J. L. Bruneel, L. Servant, L. Thouin, Electrochem. Commun., 2000, 2, 235.

6 F. Deiss, N. Sojic, D. J. White, P. R. Stoddart, Anal. Bioanal. Chem., 2011, 396, 53.

7 C.L. Brosseau, F. Casadio, R. P. Van Duyne, J. Raman Spect. 2011, 42, 1305.

8 D. P. dos Santos, M. L. A. Temperini, A. G. Brolo, J. Am. Chem. Soc., 2012, 134, 13492

9 X.Shan, U. Patel, S.Wang, R. Iglesias, N. Tao, Science 2010, 327, 1363.

10 S. Szunerits, N. Knorr, R. Calemczuk, T. Livache, Langmuir, 2004, 20, 9236.

11 S. Munteanu, N. Garraud, J. P. Roger, F. Amiot, J. Shi, Y. Chen, C. Combellas, F. Kanoufi,, Anal. Chem., $2013,85,1965$.

12 G. Wittstock, M. Burchardt, S. E. Pust, Y. Shen, C. Zhao, Angew. Chem. 2007, 119, 1604; Angew. Chem. Int. Ed. 2007, 46, 1584.

13 S. Nunige, H. Hazimeh, R. Cornut, C. Lefrou, C. Combellas, F. Kanoufi, Angew. Chem. Intl. Ed., 2012, $51,5208$.

14 D. Mandler In Scanning Electrochemical Microscopy, A.J. Bard and M.V. Mirkin Eds., CRC Press: Boca Raton, 2012, Chap 15, pp. 489-524.

15 H.V. Patten, K. E. Meadows, L. A. Hutton, J. G. Iacobini, D. Battistel, K. McKelvey, A. W. Colburn, M. E. Newton, J. V. Macpherson, P. R. Unwin, Angew. Chem., Intl Ed. 2012, 51, 7002-7006.

16 A. N. Patel, K. McKelvey, P. R. Unwin, J. Am. Chem. Soc., 2012, 134, 20246.

17 Q.G. Li, H. S. White, Anal. Chem., 1995, 67, 561.

18 O. Andersson, C. Ulrich, Bjorefors, B. Liedberg, Sens. Actuators B : Chem., 2008, 134, 545.

19 A. R. Perry, M. Peruffo, P. R. Unwin, Cryst. Growth Des., 2013, 13, 614

20 R. C. Engstrom, C. M. Pharr, M. D. Koppang, J. Electroanal. Chem., 1984, 221, 251.

21 C. Amatore, A. Chovin, P. Garrigue, L. Servant, N. Sojic, S. Szunerits, L. Thouin, Anal. Chem., 2004, 76, 7202.

22 R. G. Maus, E. M. McDonald, R. M. Wightman, Anal. Chem. 1999, 71, 4944.

23 S. Szunerits, J. M. Tam, L. Thouin, C. Amatore, D. R. Walt, Anal. Chem., 2003, 75, 4382.

24 N. Garraud, Y. Fedala, F. Kanoufi, G. Tessier, J. P. Roger, F. Amiot, Opt. Lett., 2011, 4, 594.

25 M. Born, E. Wolf, In Principles of Optics, Pergamon Press: London, 1980.

26 P. Drude, Ann. Phys. Chem., N.F., 1889, 36, 865.

27 Handbook of Optical Constants of Solids, Edward D. Palik. Academic Press: Boston, 1985.

28 M. Stedmann, Symp. Faraday Soc., 1970, 4, 64.

29 A. Laforgue, T. Addou, D. Bélanger, Langmuir, 2005, 21, 6855.

30 S. Chernyy, A. Bousquet, K. Torbensen, J. Iruthayaraj, M. Ceccato, S. U. Pedersen, K. Daasbjerg, Langmuir, $2012,28,9573$. 
31 M. Ceccato, A. Bousquet, M. Hinge, S. U. Pedersen, K. Daasbjerg, Chem. Mater., 2011, 23, 1551.

32 A. Bousquet, M. Ceccato, M. Hinge, S.U. Pedersen, K. Daasbjerg, Langmuir, 2012, 28, 1267.

33 C. Combellas, F. Kanoufi, S. Nunige, Chem. Mater., 2007, 19, 3830.

34 J. Pinson, D. Bélanger, Chem. Soc. Rev., 2011, 40, 3995.

35 C. Combellas, F. Kanoufi, J. Pinson, F. I. Podvorica, Langmuir, 2005, 21, 280

36 A. Adenier, C. Combellas, F. Kanoufi, J. Pinson, F. I. Podvorica, Chem. Mater., 2006, 18, 2021.

37 I. Bhugun, J.-M. Savéant, J. Electroanal. Chem., 1995, 395, 127.

38 H. Zollinger, Angew. Chem. Int. Ed., 1978, 17, 141 and references therein.

39 C. Galli, Chem. Rev., 1988, 88, 765.

40 J.-M. Seinberg, M. Kullapere, U. Mäeorg, F. C. Maschion, G. Maia, D. J. Schiffrin, K. Tammeveski, J. Electroanal. Chem., 2008, 624, 151.

41 I. Szele, H. Zollinger, Helv. Chim. Acta, 1978, 61, 1721.

42 K. Ishida, N. Kobori, M. Kobayashi, H. Minato, Bull. Chem. Soc. Jpn., 1970, 43, 285.

43 R. Pazo-Llorente, C. Bravo -Díaz, E. Gonzalez - Romero, Eur. J. Org. Chem., 2004, 3221.

44 M. Delamar, R. Hitmi, J. Pinson, J.-M.Savéant, J. Am. Chem. Soc., 1992, 114, 5883.

45 J.-M. Savéant, Adv. Phys. Org. Chem., 2000, 35, 117.

46 H. Hazimeh, F. Kanoufi, C. Combellas, J.-M. Mattalia, C. Marchi-Delapierre, M. Chanon, J. Phys. Chem. C, $2008,112,2545$.

47 C. Amatore, In Organic Electrochemistry: An Introduction and a Guide, 3rd ed.; M.M. Baizer, H. Lund, Eds.; M. Dekker: New York, 1991, pp 207-232.

48 C. P. Andrieux, J. Pinson, J. Am. Chem. Soc., 2003, 125, 14801.

49 H. Hazimeh, S. Nunige, R. Cornut, C. Lefrou, C. Combellas, F. Kanoufi, Anal. Chem., 2011, 83, 6106.

50 K. Torbensen, K. Malmos, F. Kanoufi, C. Combellas, S. U. Pedersen, K. Daasbjerg, ChemPhysChem, $2012,13,3303$. 